\title{
Compressive Spectrum Sensing with Temporal-Correlated Prior Knowledge Mining
}

\author{
Jianfeng Liu $\mathbb{D}$, Xin-Lin Huang, and Ping Wang $(\mathbb{D}$ \\ Department of Information and Communication Engineering, Tongji University, Shanghai 201804, China \\ Correspondence should be addressed to Ping Wang; pwang@tongji.edu.cn
}

Received 25 February 2021; Revised 16 March 2021; Accepted 27 March 2021; Published 10 April 2021

Academic Editor: Xin Liu

Copyright (C) 2021 Jianfeng Liu et al. This is an open access article distributed under the Creative Commons Attribution License, which permits unrestricted use, distribution, and reproduction in any medium, provided the original work is properly cited.

\begin{abstract}
Cognitive radio (CR) has been proposed to mitigate the spectrum scarcity issue to support heavy wireless services on sub-3GHz. Recently, broadband spectrum sensing becomes a hot topic with the help of compressive sensing technology, which will reduce the high-speed sampling rate requirement of analog-to-digital converter. This paper considers sequential compressive spectrum sensing, where the temporal correlation information between neighboring compressive sensing data will be exploited. Different from conventional compressive sensing, the previous compressive sensing data will be fused into prior knowledge in current spectrum estimation. The simulation results show that the proposed scheme can achieve $98.7 \%$ detection probability under $3.5 \%$ false alarm probability and performs the best compared with the typical BPDN and OMP schemes.
\end{abstract}

\section{Introduction}

In recent years, with the explosive growth of wireless devices, the scarcity of spectrum has become an important challenge to support high-speed services to numerous users. In this case, cognitive radio (CR) is considered as an important solution to the problem of spectrum scarcity. The concept of cognitive radio was firstly proposed in the reference [1] in the late 1990s. This technology can alleviate the contradiction between scarce spectrum resources and a large number of wireless services.

However, different researchers have different definitions of CR. Among them, the Federal Communications Commission (FFC) [2] defines CR as a radio that can change its state according to its operating radio environment. Different from traditional communication systems, CR has the characteristics of high intelligence, flexibility, and self-adaptation. CR makes decisions with the help of information collected from the RF front-end environment and can learn and analyse problems from historical information. More specifically, CR enables secondary users (SUs) to perform a series of operations as follows: (1) spectrum sensing to predict what spectrum is available and recognize the presence of the primary user (PU) when a PU reoccupies the licensed channel; (2) spectrum management to select the best available channel from the spectrum pool for special services; (3) spectrum sharing to coordinate access to all available channels with other SUs; and (4) spectrum mobility to vacate the channel as soon as possible when a PU is detected [3]. CR includes spectrum sensing, spectrum allocation, spectrum switching, spectrum mobility management, and other key technologies [4-6]. Spectrum sensing [7] is one of the most important modules, and its performance will directly affect the actions to be taken in the subsequent processing modules. One of the tasks of spectrum sensing is to detect the idle spectrum by sensing the surrounding environment. It also can detect the appearance of PUs in a timely and accurate manner.

Meanwhile, the compressive sensing (CS) theory states that as long as the signal itself or the signal in a certain transform domain is sparse, the original signal can be recovered with samples less than Nyquist samples. Due to the low spectrum utilization on sub- $3 \mathrm{GHz}$, the spectrum is usually sparse in frequency domain. Therefore, the CS technology is widely used in the signal acquisition and spectrum sensing of CR. Reference [8] applies CS theory to cognitive radio for the first time, which uses sub-Nyquist sampling rate to acquire wideband signals. Recently, lots of references discuss CR management for Internet for Things (IoT) and Industrial Internet for 
Things (IIoT). Reference [9] proposes a cluster-based cognitive Industrial Internet of Things (CIIoT) to improve sensing and transmission performance of the CIIoT. Reference [10] proposes a multibeam satellite IIoT in Ka-band to realize wide-area coverage and long-distance transmissions, which uses nonorthogonal multiple access (NOMA) for each beam to improve transmission rate. Reference [11] designs a 5Gbased IoT to transfer both $5 \mathrm{G}$ and IoT information simultaneously. Compared with the above works, this paper exploits the temporal correlation between adjacent spectrum. This paper proposes a convex optimization model to reconstruct the target spectrum by maximizing the inner product of the prior spectrum and the target spectrum and minimizing the $\ell_{1}$-norm of the target spectrum. We adopt this CS-based model in spectrum sensing, and thus, the number of sampling rate is reduced with compressive sensing technology. The innovation of this paper is to merge prior information into the process of spectrum sensing, and this method is robust to the slight disturbance in prior information. The proposed algorithm considers the time correlation between the spectrum in the previous time slot and the spectrum in the current time slot. Meanwhile, it considers the spectrum status in the previous time slot as the prior information to obtain the spectrum status in the current time slot.

The rest of this paper is organized as follows. The second section introduces the basic concepts and system model of compressive spectrum sensing. The third section introduces the algorithm flow and complexity analysis of the proposed compressive spectrum sensing algorithm. The fourth section shows the simulation results with performance analysis, which includes standardized mean square error (MSE), the probability of false alarm, and the probability of correct detection. The final section concludes this paper.

\section{Cognitive Radio Compressive Spectrum Sensing}

2.1. Compressive Sensing (CS) Theory. Compressive sensing (CS) technology includes three parts: the sparse representation of the signal, the measurement of the observation matrix, and the design of the signal reconstruction algorithm. The selection of the observation matrix must meet the Restricted Isometry Properly (RIP) [12, 13].

Compressive sensing (CS) concerns the problem of recovering a high-dimensional sparse (or nearly sparse) signal from a relatively small number of noisy measurements

$$
y=A x^{*}+n,
$$

where $x^{*} \in \mathbb{R}^{n}$ denotes the signal to be estimated, $A \in \mathbb{R}^{m \times n}$ is the measurement matrix, and $n \in \mathbb{R}^{m}$ is the observation noise vector. To reconstruct $x^{*}$, a standard approach, named Basic Pursuit (BP) [14], was proposed to use $\ell_{1}$-norm as a surrogate function to promote sparsity, that is,

$$
\min _{x}\|x\|_{1} \quad \text { s.t. } y=A x
$$

where $\|x\|_{p}=\left(\sum_{i=1}^{n}\left|x_{i}\right|^{p}\right)^{1 / p}$ denotes the standard $\ell_{p}$-norm of $x$.

When the observation vector is disturbed by noise, researchers propose an approach, namely, Basis Pursuit De Noising (BPDN) [15], that is,

$$
\min _{x}\|x\|_{1} \quad \text { s.t. }\|y-A x\|_{2} \leq \delta \text {, }
$$

where $\delta$ is the upper bound (in terms of the $\ell_{1}$-norm) of the noise vector $n$. Research shows that CS technology can be well applied to the reconstruction of sparse signals.

\subsection{Combination of Compressive Sensing and Cognitive} Radio. Nowadays, the utilization rate of broadband wireless spectrum resources is low, and there are a large number of unused "spectrum holes" [16]. Due to the low spectrum utilization on sub-3GHz, the spectrum is usually sparse in frequency domain. Therefore, CS technology can be used in spectrum sensing. With the help of CS technology, establishing a spectrum reconstruction scheme with low sampling rate is very significant for reducing the hardware complexity of spectrum sensing.

With the bandwidth of spectrum sensing becomes wider and wider, in order to sense such broadband signals, traditional sampling method requires a very high sampling rate. Applying CS in spectrum sensing can reduce the amount of sampled data while retaining useful spectrum information. The basis of CR is that at a certain moment, only few frequency bands are used by authorized users, and other valuable spectrum resources are in an idle state. In reality, the authorized users' spectrum resource utilization rate is low. It is shown in Figure 1.

In Figure 1, we can see the status of the spectrum at a certain moment. Authorized users only use a small portion of spectrum resource. In general, CS technology is to perform compressed observations on discrete signals followed by the reconstruction of the signal itself with a small number of observations, while CR spectrum compressive sensing is to perform compressed observations on PUs' signals followed by the reconstruction of the corresponding spectrum, so as to analyse the subchannel status in the spectrum. In spectrum compressive sensing $[17,18]$, cognitive users perform compressed measurements on target authorized signals and finally analyse the status of subchannels in the spectrum to determine the occupancy status of these subchannels, which requires observations for the accurate reconstruction of signal spectral density.

In many practical applications of CS, many prior information of the signal can be obtained. For example, in spectrum sensing, the spectrum in the previous time slot is very similar to the spectrum in the current time slot, so previous spectrum can be used as the prior information for current spectrum estimation. In [19], Mota et al. add the difference between the target signal and prior information as a penalty term to the objective function of the BP method, that is

$$
\min _{x}\|x\|_{1}+\lambda g(x-\phi) \quad \text { s.t. }\|y-A x\|_{2} \leq \delta
$$




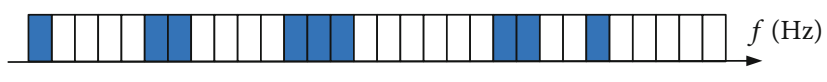

Occupied by authorized users

Not occupied by authorized users

FIGURE 1: Sparsity characteristics of spectrum resources.

where $\lambda>0$ is a tradeoff parameter and $g: \mathbb{R}^{n} \rightarrow \mathbb{R}$ is a function which measures the similarity between $x^{*}$ and $\phi$. In addition, reference [20] also analyses the following two models: $\ell_{1}-\ell_{1}$ norm minimization and $\ell_{1}-\ell_{2}$ norm minimization. That is to say, when $g(x-\phi)=\|x-\phi\|_{1}$ or $g(x-$ $\phi)=\|x-\phi\|_{2}^{2} / 2$, formula (4) can be further written as

$$
\begin{gathered}
\min _{x}\|x\|_{1}+\lambda\|x-\phi\|_{1} \quad \text { s.t. }\|y-A x\|_{2} \leq \delta, \\
\min _{x}\|x\|_{1}+\frac{\lambda}{2}\|x-\phi\|_{2}^{2} \quad \text { s.t. }\|y-A x\|_{2} \leq \delta .
\end{gathered}
$$

The above method has good performance when the prior information is sparse. But when the prior information is not sparse, the reconstruction results of the above methods are worse than the standard $\ell_{1}$-norm minimization method. Therefore, the above methods are greatly affected by small disturbances of the prior information. In order to improve the robustness of the recovery model, reference [21] proposes the following CS model, called Maximizing Correlation $(\mathrm{MC})$, that is,

$$
\min _{x}\|x\|_{1}-\lambda\langle x, \phi\rangle \quad \text { s.t. }\|y-A x\|_{2} \leq \delta
$$

where $\lambda>0$ is a tradeoff parameter. The motivation is natural because if $\phi$ is very similar to $x^{*}$, then they may be highly correlated.

Because the spectrum in the previous time slot has a high similarity to the spectrum in the current time slot in CR, the previous spectrum can be used as the prior information to estimate the current spectrum. When the prior information is good enough, model (6) can improve the performance of traditional CS technology. Therefore, in the next section, we merge model (6) into spectrum sensing.

\section{Spectrum Sensing Scheme with Temporal- Correlated Prior Knowledge}

In this section, we analyse the complexity of model (6) and compare it with other CS technology. In addition, we propose the compressive spectrum sensing scheme with temporalcorrelated prior knowledge.

For model (6), reference [22] points out a theorem: let $A \in \mathbb{R}^{m \times n}$ be a random matrix whose rows $\left\{A_{i .}\right\}_{i=1}^{m}$ are independent, centered, isotropic, and sub-Gaussian random vectors, and let $\Gamma_{f}$ denote the tangent cone of $f(x)=\|x\|_{1}-\lambda\langle x, \phi\rangle$ at $x^{*}$. If

$$
\sqrt{m} \geq C K^{2} \gamma\left(\Gamma_{f} \cap S^{n-1}\right)+\varepsilon,
$$

then with probability at least $1-2 \exp \left(-\gamma^{2}\left(\Gamma_{f} \cap S^{n-1}\right)\right)$, the solution $\hat{x}$ of (6) satisfies

$$
\left\|\widehat{x}-x^{*}\right\|_{2} \leq \frac{2 \delta}{\varepsilon},
$$

where $\varepsilon, C$ are absolute constants and $K=\max _{i}\left\|A_{i .}\right\|_{\varphi 2}$.

Reference [22] also defines the following two constants:

$$
\begin{gathered}
v_{1} \triangleq \max _{w \in \partial\left\|x^{*}\right\|_{1}-\lambda \phi}\|w\|_{2}^{2}, \\
u_{1} \triangleq\left\|\operatorname{sign}\left(x^{*}\right)-\lambda \phi\right\|_{2}^{2} .
\end{gathered}
$$

Then, according to the lemma: let $x^{*} \in \mathbb{R}^{n}$ be an $s$-sparse vector and $\phi \in \mathbb{R}^{n}$ be its prior information. Let $\Gamma_{f}$ denote the tangent cone of $f(x)=\|x\|_{1}-\lambda\langle x, \phi\rangle$ at $x^{*}$. If $0 \notin \partial\left\|x^{*}\right\|_{1}-$ $\lambda \phi$, then

$$
w^{2}\left(\Gamma_{f} \cap S^{n-1}\right) \leq \min \left\{n \cdot\left(1-\frac{n}{v_{1}} \cdot \frac{2}{\pi}\left(1-\frac{s}{n}\right)^{2}\right), s+(n-s) u_{1}\right\} .
$$

We combine formulas (7)-(10), and formula (7) can be further written as

$$
\sqrt{m} \geq C K^{2} \min \left\{\sqrt{n \cdot\left(1-\frac{n}{v_{1}} \cdot \frac{2}{\pi}\left(1-\frac{s}{n}\right)^{2}\right)}, \sqrt{s+(n-s) u_{1}}\right\}+\varepsilon .
$$

Formula (11) shows the complexity of MC. Table 1 shows the comparison between the complexity of MC and other CS methods. From Table 1, one can see that the complexity of MC does not increase much compared with the traditional CS method. Hence, it verifies the feasibility of applying the compressive sensing method based on the maximizing correlation prior information to spectrum sensing.

This paper merges Markov process and spectrum prior knowledge into spectrum sensing on the basis of model (6) and highlights the correlation of time series. We build the model (12) to dynamically sense the state of the spectrum in $\mathrm{CR}$, that is,

$$
\begin{cases}t_{1}: \min _{x_{t_{1}}}\left\|x_{t_{1}}\right\|-\lambda\left\langle x_{t_{1}}, 0\right\rangle & \text { s.t. }\left\|y_{t_{1}}-A x_{t_{1}}\right\| \leq \delta, \\ t_{2}: \min _{x_{t_{2}}}\left\|x_{t_{2}}\right\|-\lambda\left\langle x_{t_{2}}, \widehat{x}_{t_{1}}\right\rangle & \text { s.t. }\left\|y_{t_{2}}-A x_{t_{2}}\right\| \leq \delta, \\ t_{3}: \min _{x_{t_{3}}}\left\|x_{t_{3}}\right\|-\lambda\left\langle x_{t_{3}}, \widehat{x}_{t_{2}}\right\rangle & \text { s.t. }\left\|y_{t_{3}}-A x_{t_{3}}\right\| \leq \delta, \\ \vdots & \\ t_{n}: \min _{x_{t_{n}}}\left\|x_{t_{n}}\right\|-\lambda\left\langle x_{t_{n}}, \widehat{x}_{t_{n-1}}\right\rangle & \text { s.t. }\left\|y_{t_{n}}-A x_{t_{n}}\right\| \leq \delta,\end{cases}
$$

where $x_{t_{n}} \in \mathbb{R}^{n}$ denotes the true spectrum information to be estimated at $t_{n}$ moment, $\widehat{x}_{t_{n-1}} \in \mathbb{R}^{n}$ denotes the estimated 
TABLE 1: Number of samples required for different sparse signal reconstruction methods.

\begin{tabular}{lccc}
\hline Method & Objective function & Complexity & Reference \\
\hline MC & $\min _{x}\|x\|_{1}-\lambda\langle x, \phi\rangle$ & $o\left(v_{\lambda \phi} \log n\right)$ & {$[21]$} \\
BP & $\quad\|x\|_{1}$ & $o(s \log n)$ & {$[14]$} \\
$\ell_{1}-\ell_{1}$ & $\min _{x}\|x\|_{1}+\lambda\|x-\phi\|_{1}$ & $o(\bar{h} \log n)$ & {$[20]$} \\
$\ell_{1}-\ell_{2}$ & $\min _{x}\|x\|_{1}+(\lambda / 2)\|x-\phi\|_{2}^{2}$ & $o\left(v_{\lambda} \log n\right)$ & {$[20]$} \\
\hline
\end{tabular}

$v_{\lambda \phi}, s, \bar{h}$, and $v_{\lambda}$ are constant, and $n$ is the dimension of the signal to be estimated.

TABLE 2: The proposed spectrum sensing algorithm flow.

Spectrum sensing algorithm with temporal-correlated prior knowledge mining

(1) Initialize various parameters, including the number of Nyquist samples $N$, the maximum sparsity $K$, the energy decision threshold $\theta$, the number of Monte Carlo cycles $i$, the orthonormal basis $\Psi$, the observation matrix $\Phi$, and the sensing matrix $A$

(2) For the spectrum at $t_{1}$ moment, use the $\ell_{1}$-norm convex optimization model to obtain the spectrum measurement value $\widehat{x}_{t_{1}}$ at $t_{1}$ moment

(3) Consider small changes in the spectrum between $t_{1}$ moment and $t_{2}$ moment. We consider that the probability of a random spectrum change is $3 \%$ (in practice, PUs' spectrum utilization is about $15 \%$ according to the report of FCC, and thus, the spectrum state changes with a small probability. The value of such probability depends on the type of service, and the service arriving rate is usually modeled as a Poisson distribution. Hence, the reasonable spectrum change probability is less than $5 \%$ according to our experience.). The initial frequency spectrum $x_{t}$ changes slightly and becomes $\tilde{x}_{t_{1}}$, and we get a new observation value $y_{t_{2}}$ $=A \tilde{x}_{t_{1}}$

(4) When we estimate the spectrum at $t_{2}$ moment, we merge the spectrum prior information at $t_{1}$ moment. Process the prior information with the follow steps

(a) Set a threshold value $\sigma$. For each element in vector $\widehat{x}_{t_{1}}$, if the element is smaller than $\sigma$, set it to 0 . And for the other elements, keep the value unchanged

(b) Use symbolic function to process the $\widehat{x}_{t_{1}}$ which we get in (a). And set $\lambda \phi_{t_{1}}=\operatorname{sign}\left(\widehat{x}_{t_{1}}\right) / 2$

(5) According to the convex model: $\min _{x_{t_{2}}}\left\|x_{t_{2}}\right\|-\lambda\left\langle x_{t_{2}}, \phi_{t_{1}}\right\rangle$ s.t. $\left\|y_{t_{2}}-A x_{t_{2}}\right\| \leq \delta$, we can obtain the spectrum $\hat{x}_{t_{2}}$ at $t_{2}$ moment

(6) Repeat the process of step (3), step (4), and step (5) to get the spectrum information $\left\{\hat{x}_{t_{1}}, \widehat{x}_{t_{2}}, \cdots, \widehat{x}_{t_{n}}\right\}$ related to the time series $\left\{t_{1}, t_{2}, \cdots, t_{n}\right\}$

(7) According to the recovered spectrum information, we calculate the probability of false alarm, the probability of correct detection, and the standardized mean square error (MSE) of different CS algorithms and compare the performance of these CS algorithms

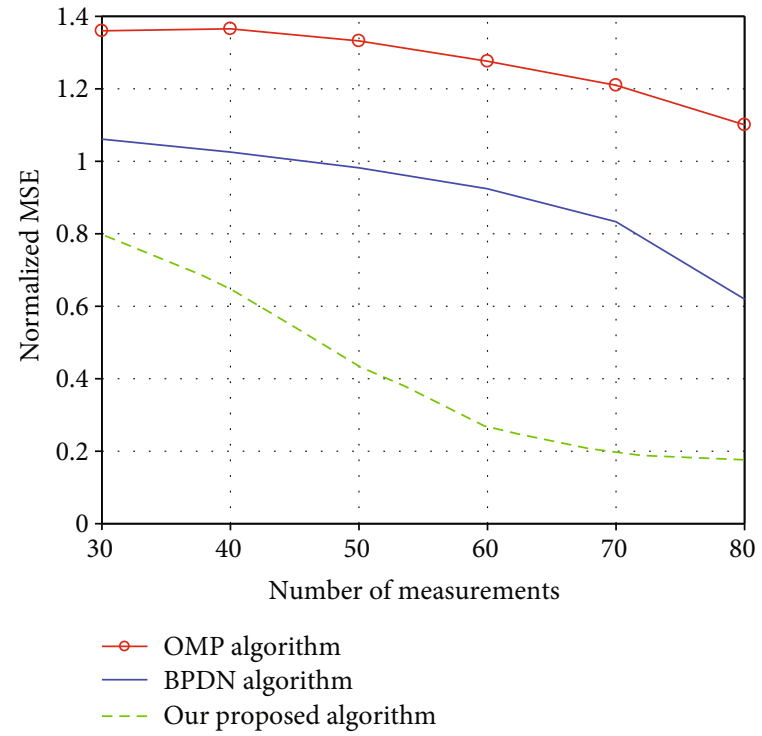

Figure 2: Performance comparisons of the reconstructed signals in terms of normalized MSE.

spectrum information at $t_{n-1}$ moment, $A \in \mathbb{R}^{m \times n}$ is the measurement matrix, $\delta$ is the upper bound (in terms of the $\ell_{2}$ -norm) of the noise vector $n$, and $y_{t} \in \mathbb{R}^{m}$ denotes the measurement vector which changes slightly over time.

The processing of the prior information largely determines the accuracy of the compressive sensing algorithm. For the prior information $\widehat{x}_{t_{n-1}}$ that obtained at $t_{n-1}$ moment, we have $\lambda \phi_{t_{n-1}}=\operatorname{sign}\left(\widehat{x}_{t_{n-1}}\right) / 2$. Therefore, model (12) can be further written as

$$
\begin{cases}t_{1}: \min _{x_{t_{1}}}\left\|x_{t_{1}}\right\|-\lambda\left\langle x_{t_{1}}, 0\right\rangle & \text { s.t. }\left\|y_{t_{1}}-A x_{t_{1}}\right\| \leq \delta, \\ t_{2}: \min _{x_{t_{2}}}\left\|x_{t_{2}}\right\|-\lambda\left\langle x_{t_{2}}, \phi_{t_{1}}\right\rangle & \text { s.t. }\left\|y_{t_{2}}-A x_{t_{2}}\right\| \leq \delta, \\ t_{3}: \min _{x_{t_{3}}}\left\|x_{t_{3}}\right\|-\lambda\left\langle x_{t_{3}}, \phi_{t_{2}}\right\rangle & \text { s.t. }\left\|y_{t_{3}}-A x_{t_{3}}\right\| \leq \delta, \\ \vdots & \\ t_{n}: \min _{x_{t_{n}}}\left\|x_{t_{n}}\right\|-\lambda\left\langle x_{t_{n}}, \phi_{t_{n-1}}\right\rangle & \text { s.t. }\left\|y_{t_{n}}-A x_{t_{n}}\right\| \leq \delta, \\ \lambda \phi_{t_{n-1}}=\operatorname{sign}\left(\widehat{x}_{t_{n-1}}\right) / 2, & \end{cases}
$$

where

$$
\operatorname{sign}(x)= \begin{cases}1, & x>0 \\ 0, & x=0 \\ -1, & x<0\end{cases}
$$

In model (13), we consider temporal correlation between neighboring compressive sensing data. According to model 


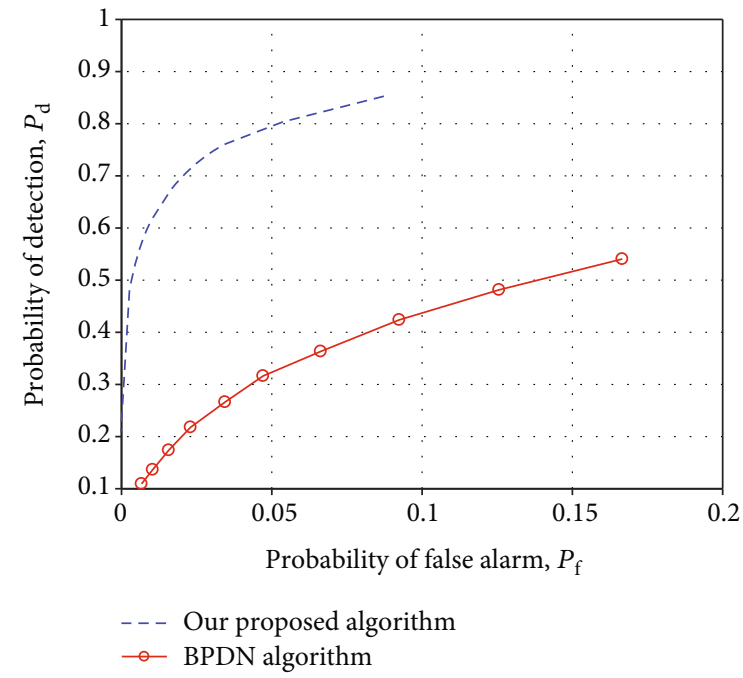

(a)

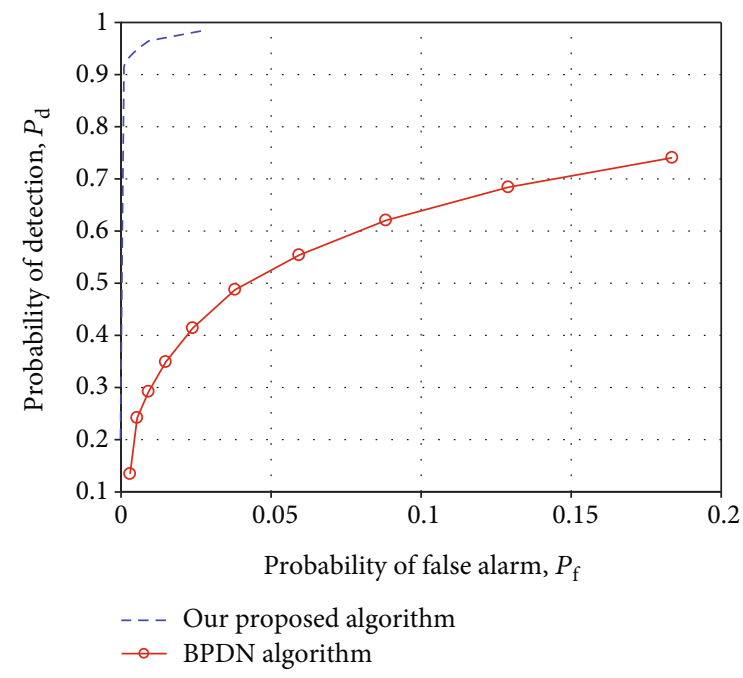

(c)

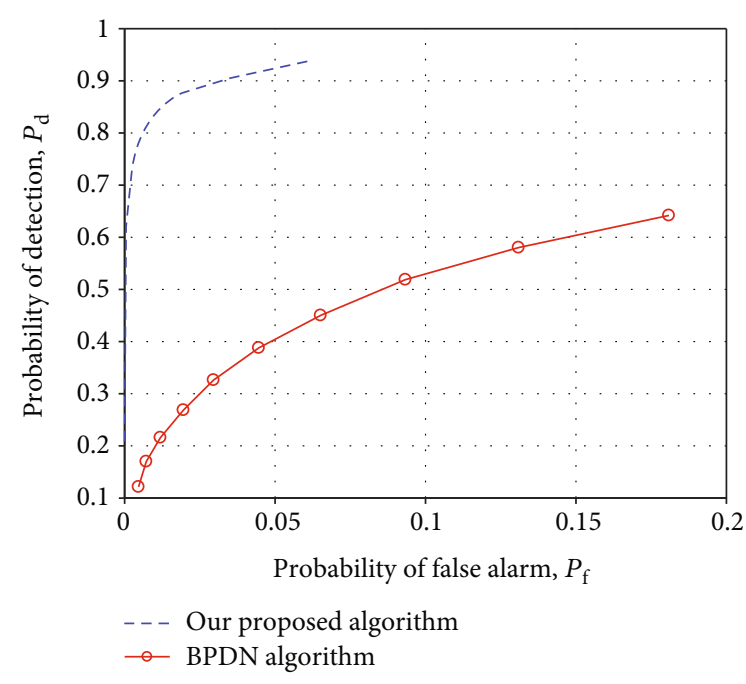

(b)

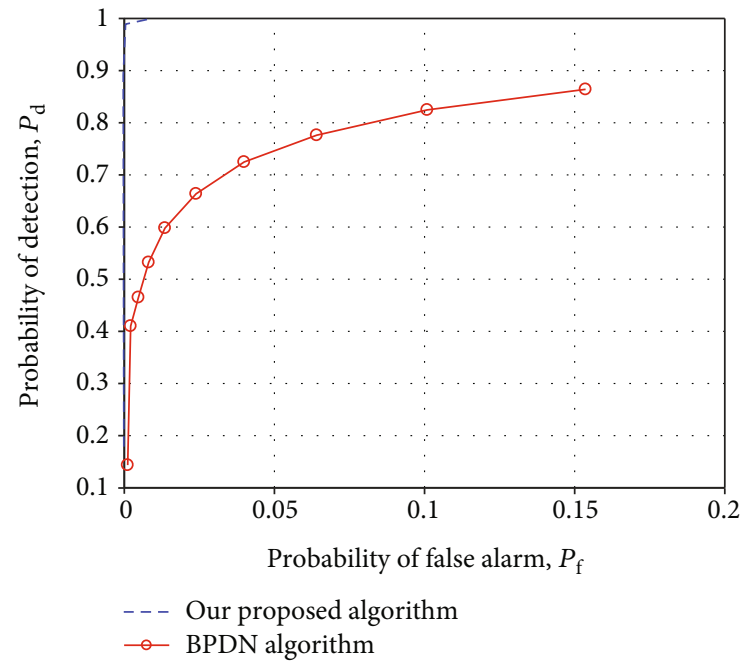

(d)

FIGURE 3: Comparisons of spectrum detection performance. Number of measurements: (a) 70. (b) 80. (c) 90. (d) 100.

(13), we propose the compressive spectrum sensing scheme with temporal-correlated prior knowledge mining. The specific steps of our proposed scheme are shown in Table 2.

\section{Performance Analysis}

In this section, we test the performance of our proposed temporal-correlated prior knowledge mining algorithm and compare it with BPDN algorithm and OMP algorithm. The $\mathrm{BPDN}$ algorithm has been introduced in the second section. The OMP algorithm is a fast greedy strategy that iteratively selects the basis function most aligned with the current residual, and its solution is based on the $\ell_{1}$-norm [23].

4.1. Performance of Reconstruction Errors. We first study the performance of spectrum map reconstruction errors during the CS inversion process in our proposed temporalcorrelated prior knowledge mining algorithm. We use the normalized MSE of the reconstructed spectrum as in [24-26], i.e.,

$$
\frac{\left\|R^{P}-\widehat{R}_{\text {reconstructed }}^{P}\right\|_{2}}{\left\|R^{P}\right\|_{2}} .
$$

For fairly comparisons, we compare the proposed algorithm with other two classical CS inversion methods (the OMP algorithm and the BPDN algorithm), and the sampling rates are set from 30 to 80 . The normalized mean square error (MSE) of the reconstructed signal is plotted in Figure 2.

From Figure 2, it can be seen that as the number of samples increases, the MSE of the three methods gradually decrease, and the performance of our proposed algorithm is better than the BPDN algorithm and the OMP algorithm. In general, compared to the traditional BPDN algorithm, 


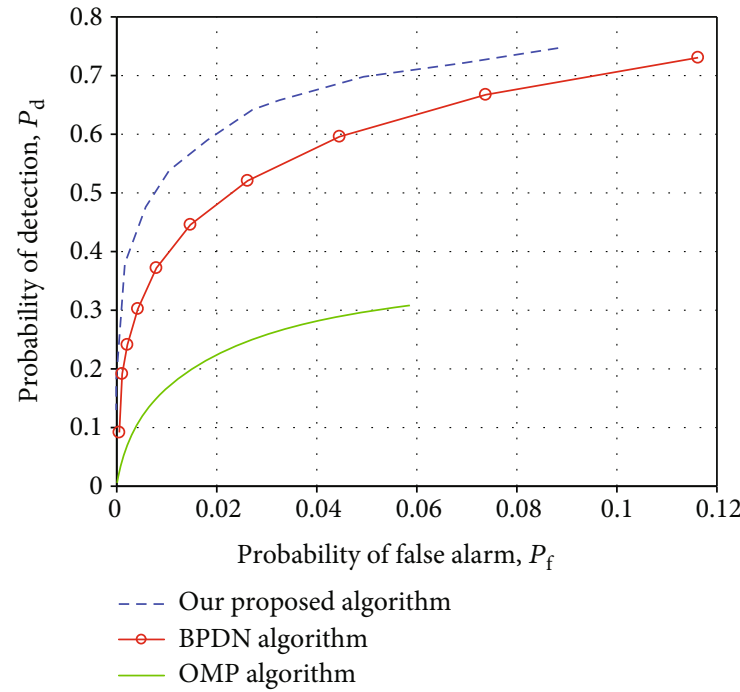

(a)

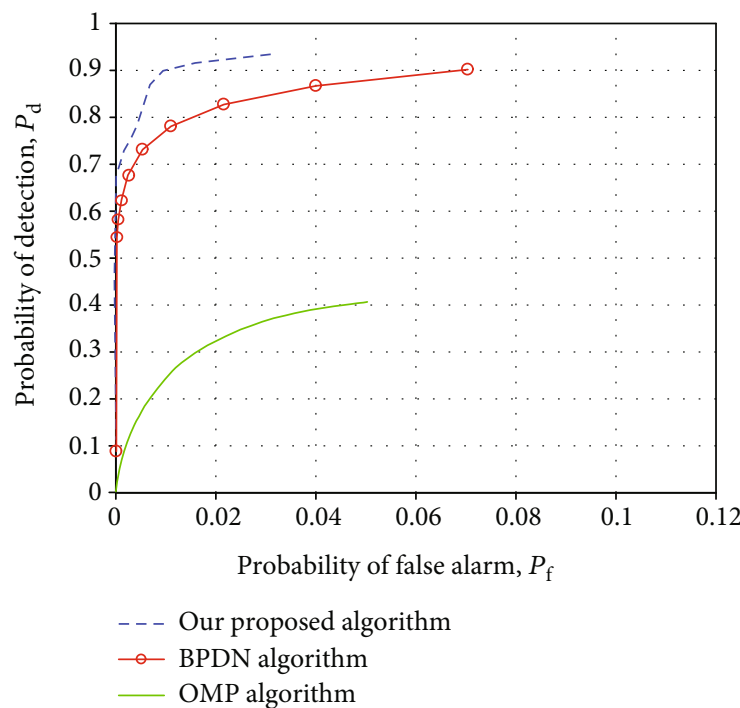

(c)

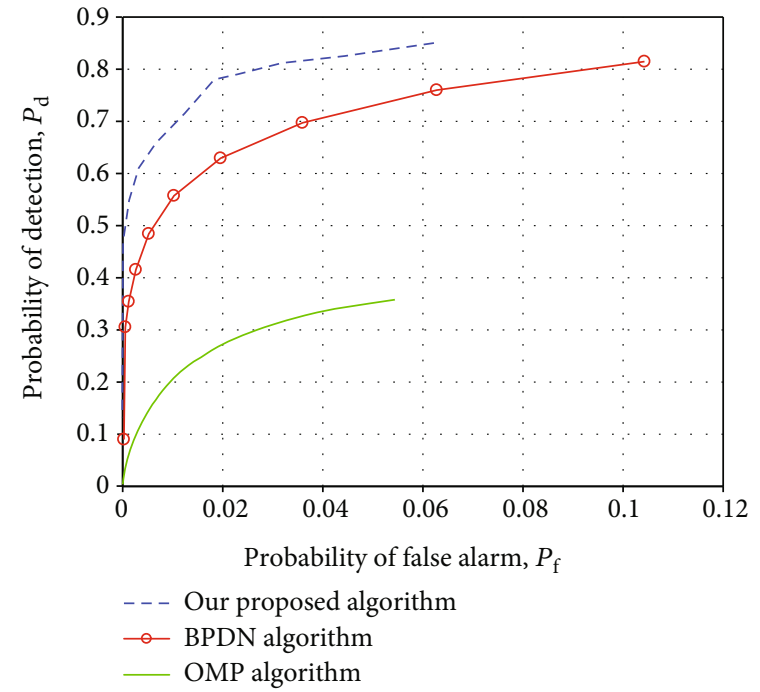

(b)

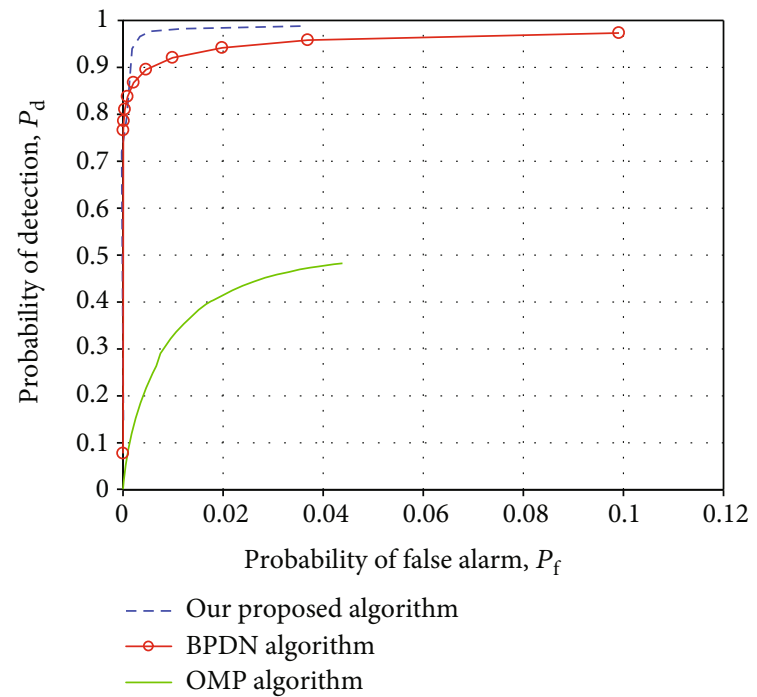

(d)

FIgURE 4: Comparisons of spectrum detection performance. Number of measurements: (a) 60. (b) 65. (c) 70. (d) 75.

the reconstruction errors of our proposed spectrum reconstruction algorithm are reduced by $20 \%$ to $60 \%$ (corresponding to 30 to 80 points of measurements, respectively) in terms of normalized MSE. This improvement is important because it means that we could achieve $60 \%$ higher accuracy of spectrum reconstruction in each SU. From Figure 2, one can see that our proposed algorithm has the smallest normalized MSE, which indicates that our scheme has better spectrumsensing performance than OMP algorithm and BPDN algorithm.

4.2. Performance of Spectrum Sensing. The main goal of spectrum sensing is to detect the characteristics of spectrum holes, and then, these detection results can be used by the spectrum decision module. Hence, here, we present the spectrum hole detection performance for our proposed algorithm. Since the key metrics in spectrum sensing are the probability of correct spectrum detection $P_{d}$ and the proba- bility of false alarm $P_{f}$ [27], we evaluate these two metrics by comparing the binary spectrum sparseness decision $\tilde{d}$ with the true subcarriers state $d$ as follows [28]:

$$
\begin{gathered}
P_{d}=E\left\{\frac{d^{T}(d=\tilde{d})}{1^{T} d}\right\}, \\
P_{f}=E\left\{\frac{(1-d)^{T}(d \neq \tilde{d})}{M-1^{T} d}\right\} .
\end{gathered}
$$

In CRN, we expect that while the spectrum sensing scheme provides a high detection probability $P_{d}$, its false alarm probability $P_{f}$ can still maintain a relatively low level. A high probability of correct detection can reduce the 


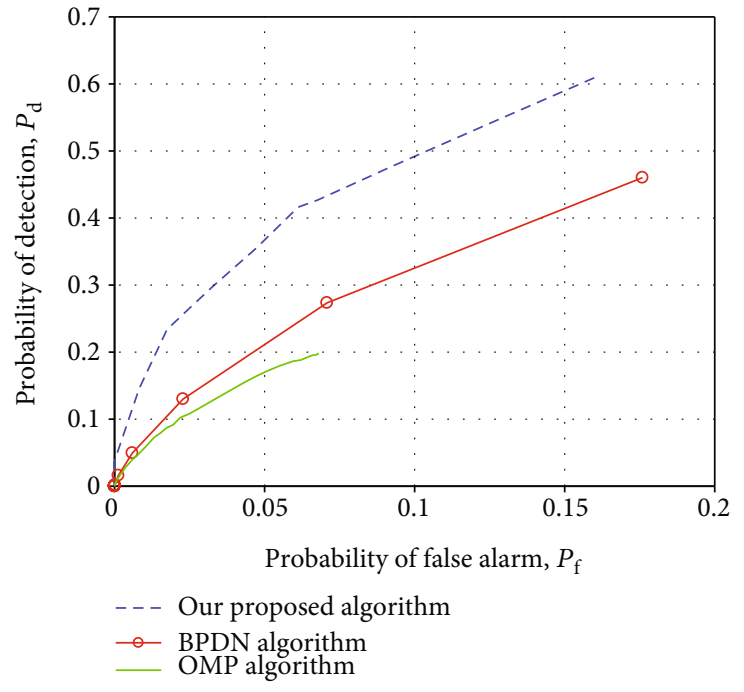

(a)

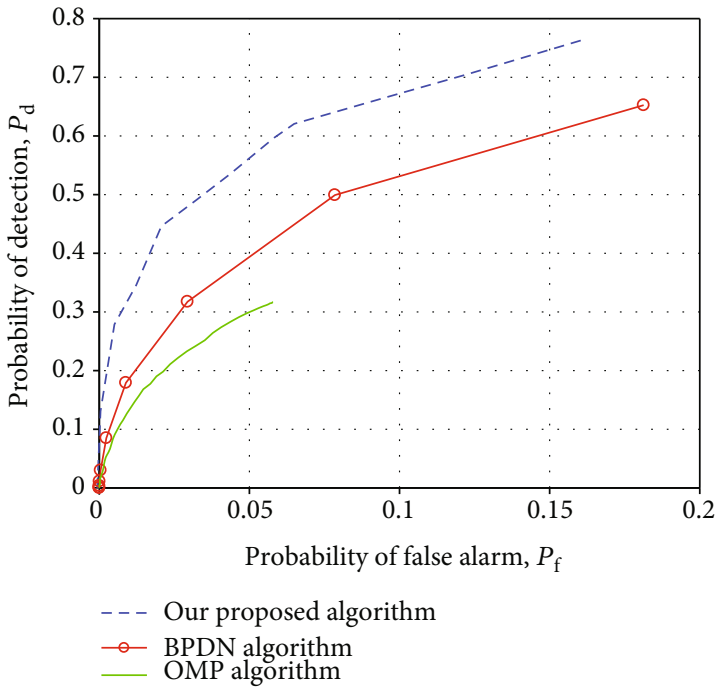

(b)

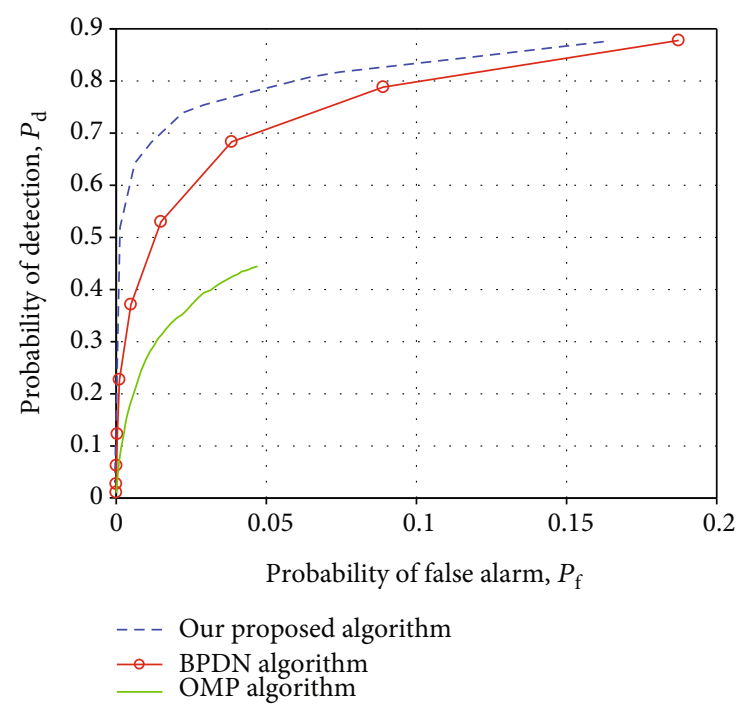

(c)

FIgURE 5: Comparisons of spectrum detection performance. SNR (dB): (a) 0. (b) 5. (c) 10.

inference to the PUs, whereas the relatively low probability of false alarm can provide more bandwidth to the SUs [27].

In the simulation, we divide a frequency spectrum into 256 subbands with equal bandwidth. The PUs occupy 35 subbands, that is, sparsity is $35 / 256$. In this simulation, we do not think of noise. First, we consider the most ideal prior information, that is $\lambda \phi=\operatorname{sign}\left(x^{*}\right) / 2$. The comparison of spectrum sensing performance is shown in Figure 3 when the number of CS observation points is $70,80,90$, and 100 .

In Figure 3, we can see that by merging the most ideal prior information into spectrum sensing, the performance of our proposed method is significantly better than the BPDN algorithm.

Next, we merge the actual prior information: $\lambda \phi_{t_{n-1}}=$ $\operatorname{sign}\left(\widehat{x}_{t_{n-1}}\right) / 2$. In this simulation, the spectrum is divided into
256 subbands with equal bandwidth. The PUs occupy 20 subbands, that is, the sparsity is $20 / 256$. We also do not think of noise. When the number of observations is $60,65,70$, and 75 , respectively, we compare the spectrum sensing performance of our proposed algorithm, the BPDN algorithm and the OMP algorithm. The simulation result is shown in Figure 4.

In Figure 4, we can see that as the number of observations increases, the performance of the BPDN algorithm and OMP algorithm gradually improves. The more measurement values obtained, the more spectrum information obtained, so we can obtain better spectrum holes detection performance. Figure 4 shows that, under the same $P_{d}$ and $P_{f}$, our proposed algorithm can further reduce the sampling rate requirements and thus has a lower sampling cost. Compared with the BPDN algorithm and the OMP algorithm, the 
performance of our proposed spectrum sensing algorithm is better.

In Figure 4, one can also see the following: (1) when the number of measurements increases, a higher probability of correct detection can be achieved since a richer spectrum information can be exploited in each SU. Meanwhile, the spectrum sensing performance curves start to deviate from the $Y$-axis more obviously (see Figure 4 ) since more spectrum information exchange among SUs also helps to reduce the possibility of false alarms. In this case, Figure 4 clearly shows that our proposed scheme has the lowest probability of false alarm among all schemes. (2) In CRN, since it is not allowed to interfere with PU, it may only be interested in the case of $P_{d}=1$. In this case (i.e., when $P_{d}=1$ ), Figure 4 shows that the probability of false alarms in our proposed algorithm is reduced by $60 \%$ compared with the BPDN algorithm. It also shows that the proposed scheme can achieve $98.7 \%$ detection probability under $3.5 \%$ false alarm probability when the number of measurements is 75 (see Figure 4(d)). This is because more spectrum holes can be accurately detected through our proposed algorithm.

Finally, on the basis of the above simulation, we merge noise into the frequency spectrum. In this simulation, the frequency spectrum is divided into 256 subbands with equal bandwidth, and the PUs occupy 20 subbands, that is, the sparsity is $20 / 256$. The number of measurements is 70 . When the signal-to-noise ratio is $0 \mathrm{~dB}, 5 \mathrm{~dB}$, and $10 \mathrm{~dB}$, respectively, we compare the spectrum sensing performance of the reconstruction algorithm with temporal-correlated prior knowledge mining, the BPDN algorithm, and the OMP algorithm. The simulation result is shown in Figure 5.

In Figure 5, one can see that as the SNR increases, the performance of the three spectrum-sensing schemes improves. In addition, it can be seen that when the SNR is small, that is, when the radio environment is poor, the advantage of our proposed spectrum sensing scheme is more obvious. In other words, our proposed scheme is more resistant to external interference.

\section{Conclusions and Future Works}

In this paper, we have proposed a compressive spectrum sensing scheme for CRN with temporal-correlated prior knowledge mining. First, we have considered the time correlation of the spectrum and merged the spectrum changes between the previous time slot and the current time slot in the spectrum sensing algorithm. Second, we have constructed a convex optimization model associated with the prior information obtained by spectrum sensing at the previous time slot to reconstruct the spectrum at the current time slot. Finally, the simulation results have demonstrated the effectiveness of our proposed spectrum sensing algorithm, which utilizes the spectrum prior knowledge at the previous time slot in CR. Our simulation results have shown that (1) compared with the BPDN algorithm and the OMP algorithm, our proposed algorithm has the smallest standardized mean square error. (2) The proposed algorithm has the best spectrum sensing performance in terms of the probability of correct detection and the probability of false alarm. Hence, the spectrum sensing scheme with temporal-correlated prior knowledge mining can be effectively applied to the spectrum sensing of CR.

In the future, we intend to focus on the multitask compressive spectrum sensing with temporal-correlated prior knowledge mining in the cluster-based CRN.

\section{Data Availability}

The data used to support the findings of this study are available from the corresponding author upon request.

\section{Conflicts of Interest}

The authors declare that they have no conflicts of interest.

\section{Acknowledgments}

This work was supported in part by the National Natural Science Foundation of China under grants 62071332, 61631017, and U1733114; in part by the Fundamental Research Funds for the Central Universities; in part by the Shanghai RisingStar Program under grant 19QA1409100; and in part by the Shanghai Science and Technology Innovation Action Plan under grant 19DZ1201100.

\section{References}

[1] J. Mitola and G. Q. Maguire, "Cognitive radio: making software radios more personal," IEEE Personal Communications, vol. 6, no. 4, pp. 13-18, 1999.

[2] FCC, Spectrum Policy Task Force Report, FCC, Washington, DC, 2002, Rep. Et Docket 02-135.

[3] X.-L. Huang, G. Wang, and F. Hu, "Multitask spectrum sensing in cognitive radio networks via spatiotemporal data mining," IEEE Transactions on Vehicular Technology, vol. 62, no. 2, pp. 809-823, 2013.

[4] A. S. Buttar, "Fundamental operations of cognitive radio: a survey," in 2019 IEEE International Conference on Electrical, Computer and Communication Technologies (ICECCT), pp. 1-5, Coimbatore, India, 2019.

[5] N. Muchandi and R. Khanai, "Cognitive radio spectrum sensing: a survey," in 2016 International Conference on Electrical, Electronics, and Optimization Techniques (ICEEOT), pp. 3233-3237, Chennai, India, 2016.

[6] F. Awin, E. Abdel-Raheem, and K. Tepe, "Blind spectrum sensing approaches for interweaved cognitive radio system: a tutorial and short course," IEEE Communications Surveys \& Tutorials, vol. 21, no. 1, pp. 238-259, 2019.

[7] A. Ali and W. Hamouda, "Advances on spectrum sensing for cognitive radio networks: theory and applications," IEEE Communications Surveys \& Tutorials, vol. 19, no. 2, pp. 1277-1304, 2017.

[8] K. Cao and H. Shao, "Compressive wideband spectrum sensing using high-order statistics for cognitive radio," in 2013 IEEE Global High Tech Congress on Electronics, pp. 187-190, Shenzhen, China, 2013.

[9] X. Liu and X. Zhang, "NOMA-based resource allocation for cluster-based cognitive industrial internet of things," IEEE Transactions on Industrial Informatics, vol. 16, no. 8, pp. 5379-5388, 2020. 
[10] X. Liu, X. B. Zhai, W. Lu, and C. Wu, "QoS-guarantee resource allocation for multibeam satellite industrial internet of things with NOMA," IEEE Transactions on Industrial Informatics, vol. 17, no. 3, pp. 2052-2061, 2021.

[11] X. Liu and X. Zhang, "Rate and energy efficiency improvements for 5G-based IoT with simultaneous transfer," IEEE Internet of Things Journal, vol. 6, no. 4, pp. 5971-5980, 2019.

[12] O. G. Uyan and V. C. Gungor, "Lifetime analysis of underwater wireless networks concerning privacy with energy harvesting and compressive sensing," in 2019 27th Signal Processing and Communications Applications Conference (SIU), pp. 1-4, Sivas, Turkey, 2019.

[13] H. Kim, J. Ahn, and H. Nam, "Wideband spectrum sensing using low-power IoT device," in 2020 International Conference on Information and Communication Technology Convergence (ICTC), pp. 424-426, Jeju, Korea (South), 2020.

[14] S. S. Chen, D. L. Donoho, and M. A. Saunders, "Atomic decomposition by basis pursuit," SIAM Review, vol. 43, no. 1 , pp. 129-159, 2001.

[15] E. J. Candes and T. Tao, "Near-optimal signal recovery from random projections: universal encoding strategies?," IEEE Transactions on Information Theory, vol. 52, no. 12, pp. 5406-5425, 2006.

[16] Y. Li and J. Wang, "Time-frequency analysis of seismic data based on Basis Pursuit," in 2014 12th International Conference on Signal Processing (ICSP), pp. 175-179, Hangzhou, China, 2014.

[17] D. L. Donoho, Y. Tsaig, I. Drori, and J. Starck, "Sparse solution of underdetermined systems of linear equations by stagewise orthogonal matching pursuit," IEEE Transactions on Information Theory, vol. 58, no. 2, pp. 1094-1121, 2012.

[18] X. Liu, M. Jia, X. Zhang, and W. Lu, "A novel multichannel internet of things based on dynamic spectrum sharing in $5 \mathrm{G}$ communication," IEEE Internet of Things Journal, vol. 6, no. 4, pp. 5962-5970, 2019.

[19] J. F. C. Mota, N. Deligiannis, and M. R. D. Rodrigues, "Compressed sensing with prior information: strategies, geometry, and bounds," IEEE Transactions on Information Theory, vol. 63, no. 7, pp. 4472-4496, 2017.

[20] H. Mansour and R. Saab, "Recovery analysis for weighted $\ell_{1}$ minimization using the null space property," Applied and Computational Harmonic Analysis, vol. 43, no. 1, pp. 23-38, 2017.

[21] X. Zhang, W. Cui, and Y. Liu, "Recovery of structured signals with prior information via maximizing correlation," IEEE Transactions on Signal Processing, vol. 66, no. 12, pp. 32963310, 2018.

[22] X. Zhang, W. Cui, and Y. Liu, "Compressed sensing with prior information via maximizing correlation," in 2017 IEEE International Symposium on Information Theory (ISIT), pp. 221225, Aachen, 2017.

[23] G. M. Salama and S. A. Taha, "Cooperative spectrum sensing and hard decision rules for cognitive radio network," in 2020 $3 r d$ International Conference on Computer Applications \& Information Security (ICCAIS), pp. 1-6, Riyadh, Saudi Arabia, 2020.

[24] S. Ji, D. Dunson, and L. Carin, "Multitask compressive sensing," IEEE Transactions on Signal Processing, vol. 57, no. 1, pp. 92-106, 2009.

[25] Y. Qi, D. Liu, D. Dunson, and L. Carin, "Multi-task compressive sensing with Dirichlet process priors," in Proceedings of the 25th International Conference on Machine Learning, pp. 768-775, Helsinki, Finland, 2008.

[26] J. A. Bazerque and G. B. Giannakis, "Distributed spectrum sensing for cognitive radio networks by exploiting sparsity," IEEE Transactions on Signal Processing, vol. 58, no. 3, pp. 1847-1862, 2010.

[27] X.-L. Huang, G. Wang, F. Hu, and S. Kumar, "The impact of spectrum sensing frequency and packet-loading scheme on multimedia transmission over cognitive radio networks," IEEE Transactions on Multimedia, vol. 13, no. 4, pp. 748-761, 2011.

[28] F. Zeng, C. Li, and Z. Tian, "Distributed compressive spectrum sensing in cooperative multihop cognitive networks," IEEE Journal of Selected Topics in Signal Processing, vol. 5, no. 1, pp. 37-48, 2011. 